\title{
Borromean surgery formula for the Casson invariant
}

\author{
JEAN-BAPTISTE MEILHAN
}

\begin{abstract}
It is known that every oriented integral homology 3-sphere can be obtained from $S^{3}$ by a finite sequence of Borromean surgeries. We give an explicit formula for the variation of the Casson invariant under such a surgery move. The formula involves simple classical invariants, namely the framing, linking number and Milnor's triple linking number. A more general statement, for $n$ independent Borromean surgeries, is also provided.
\end{abstract}

$57 \mathrm{~N} 10,57 \mathrm{M} 27$

\section{Introduction}

A Borromean surgery link in a closed oriented 3-manifold $M$ is an oriented framed link obtained by embedding in $M$ a copy of the standard genus 3 handlebody containing the 6-component 0 -framed oriented link depicted below. (Here and throughout this paper, blackboard framing convention is used.) Surgery along such a link is called a Borromean surgery move.

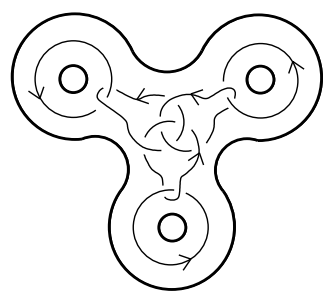

The notion of Borromean surgery was first introduced by Matveev [9] in the eighties, and was more recently used to define the Goussarov-Habiro finite type invariant theory for closed oriented 3-manifolds [2; 4].

Matveev showed that two closed oriented 3-manifolds are Borromean equivalent, ie are related by a sequence of Borromean surgery moves, if and only if they have the same first homology groups and isomorphic linking forms. In particular, any oriented integral homology 3-sphere is obtained from $S^{3}$ by a finite sequence of Borromean surgeries. It is thus a natural problem to study the behavior of classical invariants of 
integral homology spheres, such as the Casson invariant, under this kind of surgery move. Our main result is Theorem 2.2, which expresses the variation of the Casson invariant under a Borromean surgery move in terms of simple classical invariants of the Borromean surgery link, namely framings, linking numbers and Milnor's triple linking number - see Section 2.3 for an explicit statement. A more general formula, for $n$ independent Borromean surgeries on an integral homology sphere, is also provided in Theorem 2.3, which involves an additional cubic expression in some linking numbers of the surgery link.

The paper is organized as follows. In Section 2, we recall the definition and basic properties of the Casson invariant and state the above-mentioned surgery formulas. In Section 3 we prove a couple of preliminary results on Borromean surgery in $S^{3}$ using a crossing change formula for the Casson invariant due to Johannes [5]. In Section 4 we prove the formula of Theorem 2.2 for a single Borromean surgery using the results of Section 3 and Lescop's sum formula for the Casson invariant [7]. In Section 5 we use the theory of finite type invariants to prove the formula of Theorem 2.3 for multiple Borromean surgeries. In Section 6 we compare Theorem 2.2 with a similar formula derived from Lescop's global surgery formula, and we apply it to a realization problem for homology spheres of Mazur type.

Acknowledgments This paper benefited greatly from discussions with Christine Lescop at various stages of its writing. The author also thanks Kazuo Habiro, Gwénaël Massuyeau and Alex James Bene for helpful comments, and Christian Blanchet for suggesting working on this subject.

\section{Variation of the Casson invariant under Borromean surgery}

\subsection{The Casson invariant of integral homology spheres}

In this paper, an integral homology sphere will always mean an oriented integral homology 3-sphere. We denote by $\mathbf{Z} H S$ the set of integral homology spheres, considered up to orientation-preserving diffeomorphisms.

Theorem 2.1 (Casson) There exists a unique function

$$
\lambda: \mathbf{Z H S} \longrightarrow \mathbf{Z}
$$

such that, for every $M, N \in \mathbf{Z} H S$ and for every knot $K$ in $M$ :

(1) $\lambda\left(S^{3}\right)=0$. 
(2) For $n \in \mathbf{Z}$, let $M_{K_{n}}$ be the result of $(1 / n)$-Dehn surgery on $M$ along $K$. Then:

$$
\lambda\left(M_{K_{n+1}}\right)-\lambda\left(M_{K_{n}}\right)=\frac{1}{2} \Delta_{K}^{\prime \prime}(1),
$$

where $\Delta_{K}(t)$ denotes the Alexander polynomial of $K$.

Furthermore,

(3) $\lambda(M \sharp N)=\lambda(M)+\lambda(N)$, where $\sharp$ denotes the connected sum.

(4) The Rochlin invariant $\mu$ is the $\bmod 2$ reduction of $\lambda$. More precisely, $\mu(M) \equiv$ $\lambda(M)(\bmod 2)$.

This unique function is called the Casson invariant of integral homology spheres. This invariant counts, in some sense, the conjugacy classes of irreducible representations of $\pi_{1}(M)$ in $S U(2)$.

In [12], Walker extended the Casson invariant to a $\mathbf{Q}$-valued invariant of rational homology spheres, called the Casson-Walker invariant. In this paper, we will use the following normalization of the Casson-Walker invariant

$$
\lambda=\frac{\lambda_{W}}{2},
$$

where $\lambda_{W}$ denotes the normalization adopted by Walker in [12]. (Note that our notation are consistent, in the sense that if we restrict to integral homology spheres then $\lambda_{W} / 2$ coincides with Casson's original invariant $\lambda$.)

The Casson-Walker invariant was extended by Lescop to all closed oriented 3-manifolds in [6].

\subsection{Conventions}

Let $L$ be a Borromean surgery link in a rational homology sphere $M$.

Up to isotopy one can always assume that there is a 3-ball $B$ in $M$ which intersects $L$ as depicted in Figure 1. The boundary of $B$ intersects three components of $L$, called the leaves of $L$. We fix an order on the set of the leaves of $L$, and we denote them by $F_{1}, F_{2}$ and $F_{3}$ according to this order. Denote by $f_{i}$ the framing of $F_{i}$, and by $l_{i j}$ the linking number $\operatorname{lk}\left(F_{i}, F_{j}\right), 1 \leq i \neq j \leq 3$.

Observe that there is a canonical 3-component algebraically split link $F_{0}$ associated to the Borromean surgery link $L$ as follows. For each pair $1 \leq i<j \leq 3$, consider the $(2,2)$-tangle $B_{i j}$ obtained by stacking $\left|l_{i j}\right|$ copies of the clasp-shaped $(2,2)$-tangle $T_{ \pm}$depicted on the right-hand side of Figure 2 (depending on the sign of $l_{i j}$ ). If $l_{i j}=0$, 


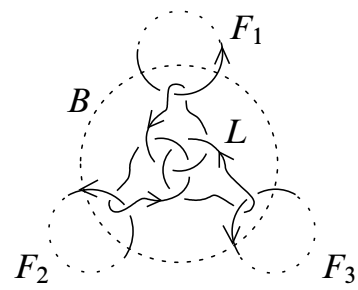

Figure 1

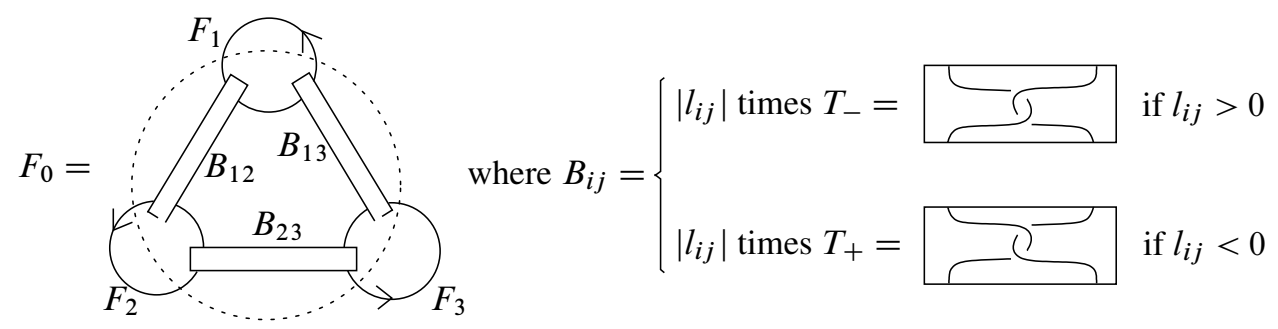

Figure 2: The algebraically split link $F_{0}$

$B_{i j}$ is the trivial $(2,2)$-tangle. Starting with the link $F_{1} \cup F_{2} \cup F_{3}$, insert for each $1 \leq i<j \leq 3$ the tangle $B_{i j}$ into the leaves $F_{i}$ and $F_{j}$ so that their linking number is reduced to zero; see Figure 2. The resulting 3-component link is the algebraically split link $F_{0}$ associated to $L$.

Because $F_{0}$ has linking numbers zero, it has a well-defined Milnor's triple linking number $\bar{\mu}_{i j k}\left(F_{0}\right)$ in $M$ (a short definition is given in Section 2.4 at the end of this section). In the following, we denote by $\mu_{123}(L ; M)$ the triple linking number of the algebraically split link $F_{0}$ associated to $L$ in $M$. We will usually simply write $\mu_{123}(L)$ when $M$ is explicit from the context.

\subsection{Statement of the main results}

We can now state our two main results.

We first express the variation of the Casson-Walker invariant under surgery along a Borromean surgery link $L$ in a rational homology sphere $M$, under certain restrictions.

Theorem 2.2 Let $L$ be a Borromean surgery link in a rational homology sphere $M$, such that the leaves of $L$ have integral linking numbers and have self-linking numbers 
zero in $\mathbf{Q} / \mathbf{Z}$. Denote by $M_{L}$ the result of surgery on $M$ along $L$. Then the difference $\lambda\left(M_{L}\right)-\lambda(M)$ is given by the formula

$$
-f_{1} \cdot f_{2} \cdot f_{3}-2 \cdot l_{12} \cdot l_{13} \cdot l_{23}-2 \cdot \mu_{123}(L)+\sum_{\circlearrowleft_{1,2,3}} l_{23} \cdot\left(l_{23}+1\right) \cdot f_{1},
$$

where the sum is over all cyclic permutations of the indices $(1,2,3)$.

Let us now consider the case of a disjoint union $L_{1} \cup \ldots \cup L_{n}$ of $n$ Borromean surgery links in an integral homology sphere $M$. We use the conventions of Section 2.2 for the orientation and ordering of the leaves of each link: the leaves of $L_{k}$ are denoted by $F_{1}^{k}, F_{2}^{k}$ and $F_{3}^{k}(1 \leq k \leq n)$. Denote by $l_{i j}^{k l}$ the linking number $\operatorname{lk}\left(F_{i}^{k}, F_{j}^{l}\right)$; $1 \leq i \neq j \leq 3,1 \leq k \neq l \leq n$.

Theorem 2.3 The difference $\lambda\left(M_{L_{1} \cup \ldots \cup L_{n}}\right)-\lambda(M)$ is given by the formula

$$
\sum_{1 \leq i \leq n}\left(\lambda\left(M_{L_{i}}\right)-\lambda(M)\right)-2 \sum_{1 \leq k<l \leq n} \sum_{\sigma \in S_{3}} s(\sigma) l_{1 \sigma(1)}^{k l} l_{2 \sigma(2)}^{k l} l_{3 \sigma(3)}^{k l}
$$

where, for an element $\sigma$ in the symmetric group $S_{3}, s(\sigma) \in\{-1 ;+1\}$ denotes its signature.

By reducing the formula in Theorem 2.3 modulo 2 and using Theorem 2.1(4), we obtain a similar formula for the variation of Rochlin's $\mu$-invariant of an integral homology sphere $M$ under surgery along a disjoint union $L_{1} \cup \ldots \cup L_{n}$ of Borromean surgery links.

\section{Corollary 2.4}

$$
\mu\left(M_{L_{1} \cup \ldots \cup L_{n}}\right)-\mu(M)=\sum_{1 \leq i \leq n} f_{1}^{i} \cdot f_{2}^{i} \cdot f_{3}^{i}(\bmod 2) .
$$

This formula was already (essentially) established independently by Massuyeau in [8], and Auclair and Lescop in [1].

\subsection{Milnor's triple linking number}

Let $K=K_{1} \cup K_{2} \cup K_{3}$ be an algebraically split, oriented, ordered link in a rational homology sphere $M$. Pick a triple $\left(S_{1}, S_{2}, S_{3}\right)$ of transverse Seifert surfaces such that each $S_{i}$ is bounded by $K_{i}(i=1,2,3)$ and does not intersect the two other components of $K$ (this is possible since all linking numbers are zero). Then Milnor's triple linking number $\bar{\mu}_{123}(K ; M)$ of $K$ in $M$ is defined as the negative of the algebraic intersection $\left\langle S_{1}, S_{2}, S_{3}\right\rangle_{M}$, defined as follows. 
Each surface $S_{i}$ is equipped with a positive normal vector field, induced by its orientation and the orientation of $M$, and thus each triple intersection point $x \in S_{1} \cap S_{2} \cap S_{3}$ can be associated a sign, depending on whether the ordered basis of normal vectors to $S_{1}, S_{2}$ and $S_{3}$ at $x$ agrees or not with the orientation of $M$. The algebraic intersection $\left\langle S_{1}, S_{2}, S_{3}\right\rangle_{M}$ is the sum of these signs over all $x \in S_{1} \cap S_{2} \cap S_{3}$.

\section{Preliminary results: Borromean surgery in $S^{3}$}

In this section we prove a couple of preliminary lemmas on Borromean surgery in $S^{3}$.

\subsection{Main tools}

We recall here a self-crossing change formula for the Casson invariant due to Johannes, as well as a simple result derived from Kirby calculus.

3.1.1 Johannes' formula for the Casson invariant In [5], Johannes expresses the difference of the Casson invariants of 3-manifolds presented by links in $S^{3}$ which differ by a crossing change within a component. This type of move on surgery links was studied by Matveev [9] under the name of Whitehead surgery.

For the purpose of the present paper, it is enough to consider the case of 2-component surgery links. Let $L^{+}=L_{1}^{+} \cup L_{2}$ and $L^{-}=L_{1}^{-} \cup L_{2}$ be two framed links in $S^{3}$, with same framing, which only differ by a crossing change on the first component. Let $L_{a} \cup L_{b}$ be the 2 -component link obtained from $L_{1}^{ \pm}$by smoothing this crossing (see Figure 3).
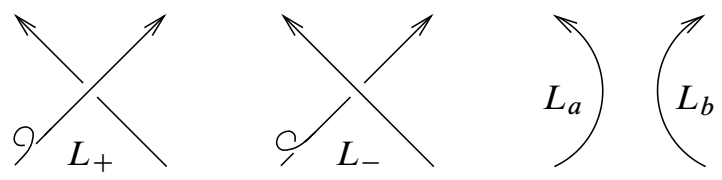

Figure 3

Then Johannes' formula [5] states that

$$
\lambda\left(S_{L^{+}}^{3}\right)-\lambda\left(S_{L^{-}}^{3}\right)=\frac{f_{2} \cdot l_{a b}-l_{a 2} \cdot l_{b 2}}{f_{1} \cdot f_{2}-l_{12}^{2}},
$$

where $f_{1}$ (resp. $f_{2}$ ) denotes the framing of $L_{1}^{ \pm}$(resp. $L_{2}$ ), and $l_{a b}$ (resp. $l_{a 2}, l_{b 2}, l_{12}$ ) denotes the linking number $\operatorname{lk}\left(L_{a}, L_{b}\right)\left(\operatorname{resp} . \operatorname{lk}\left(L_{a}, L_{2}\right), \operatorname{lk}\left(L_{b}, L_{2}\right), \operatorname{lk}\left(L_{1}^{ \pm}, L_{2}\right)\right)$. 
Observe that the denominator in (3-1) is merely the determinant of the linking matrix of $L^{ \pm}$.

Note that the formulas given in [5] are for the Casson-Walker invariant, using Walker's normalization (see Section 2.1). Note also that we fixed a sign error in [5] so that (3-1) agrees with Theorem 2.1.

Remark 3.1 Surgery along a \pm 1 -framed knot and Whitehead surgery are two kinds of surgery moves relating all integral homology spheres, and Casson and Johannes respectively gave a formula for the variation of the Casson invariant under these moves. In this paper, we give such a formula for a third type of move which relates all integral homology spheres, namely Borromean surgery.

3.1.2 Borromean surgery link and Kirby equivalence In addition to Johannes' formula, we will need the following lemma, which follows directly from several applications of Kirby moves (see [2; 4]).

Lemma 3.2 Let $K$ be a Borromean surgery link. $K$ is Kirby-equivalent to the 2component link $\widetilde{K}$ represented in Figure 4.
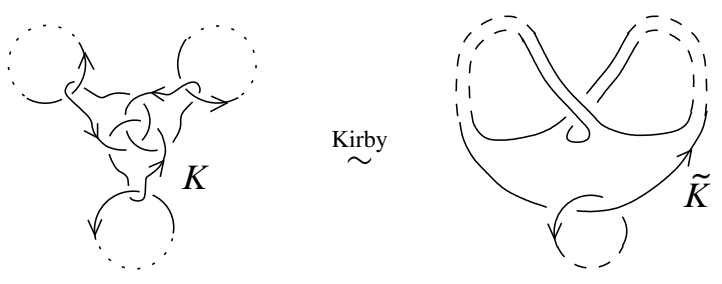

Figure 4: Two Kirby-equivalent links

Observe that the linking number of $\widetilde{K}$ is 1 , and that one of its component has always framing 0 . The determinant of the linking matrix of $\widetilde{K}$ is thus always -1 .

\subsection{Two lemmas in $S^{3}$}

We can now establish the two above-mentioned preliminary lemmas. In both statements, we consider a local operation on a Borromean surgery link in $S^{3}$, and give a formula for the variation of the Casson invariant of the surgered manifolds.

\footnotetext{
${ }^{1}$ For example, consider the right-handed trefoil knot $T$ in $S^{3}$. It can be easily verified by Theorem 2.1(2) that $\lambda\left(S_{T_{+1}}^{3}\right)=1$, but [5] would give -1 .
} 
3.2.1 Changing the framing We first consider the operation of adding a kink to a leaf of a Borromean surgery link.

Lemma 3.3 Let $K$ be a Borromean surgery link in $S^{3}$, and let $K_{i}$ be obtained by changing the framing of the $i-$ th leaf of $K$ by +1 as depicted below.
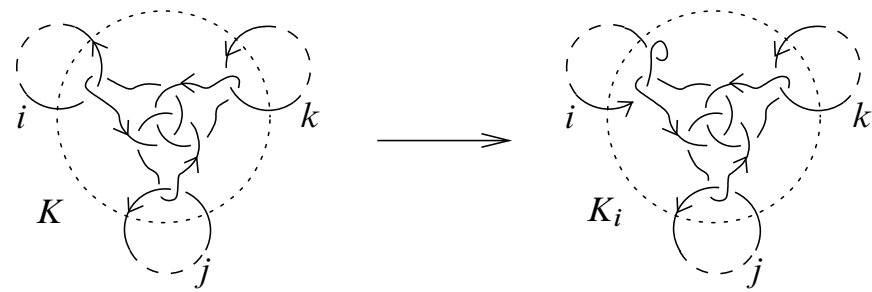

We have:

$$
\lambda\left(S_{K}^{3}\right)=\lambda\left(S_{K_{i}}^{3}\right)-f_{j} \cdot f_{k}+l_{j k}\left(1+l_{j k}\right) .
$$

Proof By Lemma 3.2, we have

$$
\lambda\left(S_{K}^{3}\right)=\lambda\left(S_{\widetilde{K}}^{3}\right) \quad \text { and } \quad \lambda\left(S_{K_{i}}^{3}\right)=\lambda\left(S_{\widetilde{K}_{i}}^{3}\right),
$$

where $\tilde{K}$ and $\widetilde{K}_{i}$ are two 2-component links as depicted in Figure 5. Consider the
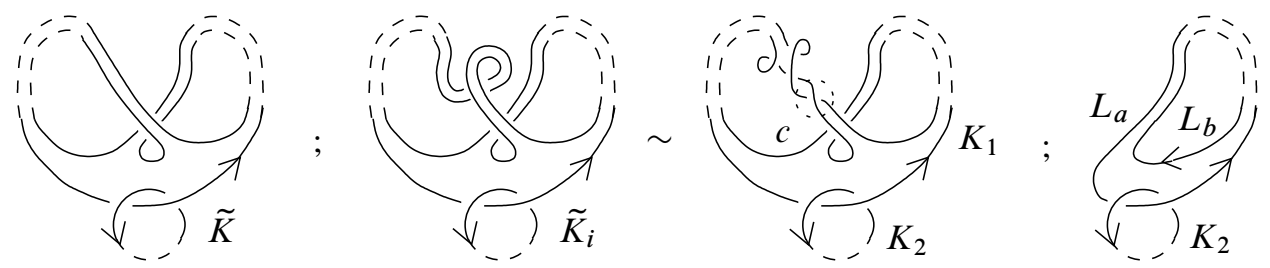

Figure 5

2-component link $K_{1} \cup K_{2}$ isotopic to $\tilde{K}_{i}$, and the positive crossing $c$ in $K_{1} \cup K_{2}$ as in Figure 5. Changing this crossing (without changing the framing) gives the link $\widetilde{K}$, and smoothing this crossing turns $K_{1}$ into a 2-component link $L_{a} \cup L_{b}$ satisfying $\operatorname{lk}\left(L_{a}, L_{b}\right)=-f_{k}, \operatorname{lk}\left(L_{a}, K_{2}\right)=1+l_{j k}$ and $\operatorname{lk}\left(L_{b}, K_{2}\right)=-l_{j k}$. The result then follows from Johannes' formula.

3.2.2 Changing the linking numbers We now study the effect, at the level of the Casson invariant, of adding a clasp between two leaves of a Borromean surgery link.

Lemma 3.4 Let $K$ be a Borromean surgery link in $S^{3}$, and let $K^{-}$be obtained by adding a clasp between the leaves $i$ and $j$ of $K$ as shown below. 


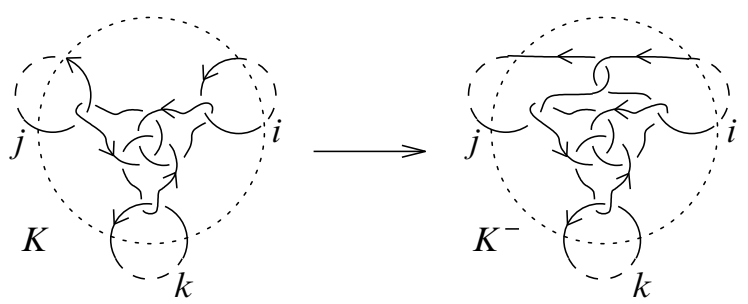

We have:

$$
\lambda\left(S_{K}^{3}\right)=\lambda\left(S_{K^{-}}^{3}\right)-2 . l_{i k} \cdot l_{j k}+2 \cdot f_{k} \cdot l_{i j} .
$$

Proof By Lemma 3.2, we have

$$
\lambda\left(S_{K}^{3}\right)=\lambda\left(S_{\widetilde{K}^{3}}^{3}\right) \quad \text { and } \quad \lambda\left(S_{K^{-}}^{3}\right)=\lambda\left(S_{\widetilde{K}^{-}}^{3}\right),
$$

where $\widetilde{K}$ and $\widetilde{K}^{-}$are two 2-component links as depicted in Figures 5 and 6 respectively. Now observe that $\widetilde{K}^{-}$is isotopic to the link $K_{1} \cup K_{2}$ of Figure 6, which only differs from $\widetilde{K}$ by four crossing changes within the component $K_{1}$. The result thus follows
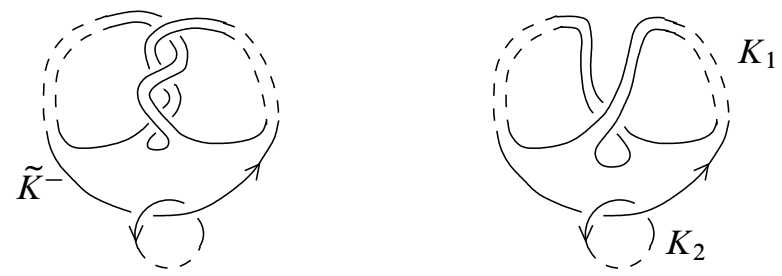

Figure 6

from 4 applications of Johannes' formula, similarly to the previous proof.

\section{Proof of Theorem 2.2}

We can now prove Theorem 2.2. Our main tool here is Lescop's sum formula for the Casson invariant [7], which we briefly review in the next subsection.

\subsection{Lescop's sum formula}

Given a compact 3-manifold $M$ with boundary, we denote by $\mathcal{L}_{M}$ the kernel of the map $H_{1}(\partial M ; \mathbf{Q}) \rightarrow H_{1}(M ; \mathbf{Q})$ induced by the inclusion. We call $\mathcal{L}_{M}$ the Lagrangian of $M$.

Let $\Sigma$ be a closed oriented surface of genus $g$, and let $A, B, A^{\prime}, B^{\prime}$ be four rational homology handlebodies such that $\partial A=\partial A^{\prime}=-\partial B=-\partial B^{\prime}=\Sigma$. Assume that in 
$H_{1}(\Sigma ; \mathbf{Q})$ we have $\mathcal{L}_{A}=\mathcal{L}_{A^{\prime}}, \mathcal{L}_{B}=\mathcal{L}_{B^{\prime}}$, and $\mathcal{L}_{A} \cap \mathcal{L}_{B}=0$. Then the main result of [7] states that $\lambda(A \cup B)-\lambda\left(A^{\prime} \cup B\right)-\lambda\left(A \cup B^{\prime}\right)+\lambda\left(A^{\prime} \cup B^{\prime}\right)$ is given by

$$
-2 \sum_{\{i, j, k\} \subset\{1, \ldots, g\}} \mathcal{I}_{A A^{\prime}}\left(\alpha_{i} \wedge \alpha_{j} \wedge \alpha_{k}\right) \mathcal{I}_{B B^{\prime}}\left(\beta_{i} \wedge \beta_{j} \wedge \beta_{k}\right),
$$

where $\left\{\alpha_{i}\right\}_{i}$, resp. $\left\{\beta_{i}\right\}_{i}$, is a basis for $\mathcal{L}_{A}$, resp. $\mathcal{L}_{B}$, such that the intersection form $\cdot$ on $\Sigma$ satisfies $\alpha_{i} \cdot \beta_{j}=\delta_{i j}$, and where $\mathcal{I}_{A A^{\prime}}$ is the intersection form on $\Lambda^{3} \mathcal{L}_{A}$ defined as follows. There is an isomorphism $H_{2}\left(A \cup_{\Sigma}-A^{\prime} ; \mathbf{Q}\right) \rightarrow \mathcal{L}_{A}$ coming from the MayerVietoris sequence which, to the homology class of an oriented surface $S \subset A \cup_{\Sigma}-A^{\prime}$, associates the class of the (oriented) boundary of $S \cap A$. Then $\mathcal{I}_{A A^{\prime}}$ is the trilinear alternating form on $\mathcal{L}_{A}$ induced, via this isomorphism, by the triple intersection form on $H_{2}\left(A \cup \cup_{\Sigma}-A^{\prime} ; \mathbf{Q}\right)$.

Note that the above sum formula is for the Casson-Walker invariant, with the normalization specified in Section 2.1.

\subsection{Proof of Theorem 2.2}

Let $L$ be a Borromean surgery link in a rational homology sphere $M$ such that the linking numbers of the leaves are integers $l_{i j}$, and such that the leaves have selflinking numbers zero in $\mathbf{Q} / \mathbf{Z}$, and thus have integral framings $f_{i}$. We aim to compute $\lambda\left(M_{L}\right)-\lambda(M)$.

Consider the Borromean surgery link $L_{\text {st }}$ of Figure 7 in the genus three handlebody $A$, standardly embedded in $S^{3}$. Denote by $B$ the exterior of $A$ in $S^{3}$, and set $A^{\prime}=A_{L_{\mathrm{st}}}$. The link $L$ can be regarded as obtained by an embedding of $A$ in $M$ such that the

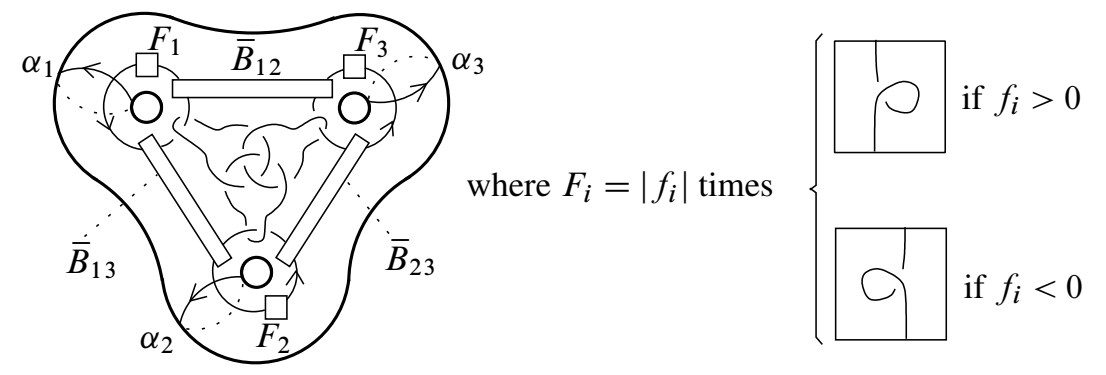

Figure 7: The link $L_{\mathrm{st}}$ in the handlebody $A$. Here $\bar{B}_{i j}$ is $\left|l_{i j}\right|$ times the $(2,2)$-tangle $T_{+}$(resp. $T_{-}$) of Figure 2 if $l_{i j}>0$ (resp. $l_{i j}<0$ ).

Lagrangians of $B$ and $B^{\prime}=M \backslash \operatorname{Int}(A)$ (ie the curves of $\partial A$ that bound in $B$ and in 
$\left.B^{\prime}\right)$ are the same. The main theorem of [7] gives

$$
\lambda\left(S^{3}\right)-\lambda\left(S_{L_{\mathrm{st}}}^{3}\right)-\lambda(M)+\lambda\left(M_{L}\right)=-2 \mathcal{I}_{A A^{\prime}}\left(\alpha_{1} \wedge \alpha_{2} \wedge \alpha_{3}\right) \mathcal{I}_{B B^{\prime}}\left(\beta_{1} \wedge \beta_{2} \wedge \beta_{3}\right),
$$

where the $\alpha_{i}$ are meridians of the three leaves of $L_{\text {st }}$ (see Figure 7), and where the $\beta_{i}$ are longitudes of the three leaves of the link $F_{0}$ of Figure 2.

For $i=1,2,3$, let $\Sigma_{B}^{i}$ (resp. $\Sigma_{B^{\prime}}^{i}$ ) be oriented surfaces in $B$ (resp. $B^{\prime}$ ) bounded by $\beta_{i}$, such that all surfaces are transverse to each other and to $\partial B$ in $B \cup_{\partial B}-B^{\prime}$. Denote by $\Sigma_{i}$ the closed surface $\Sigma_{B}^{i} \cup_{\beta_{i}} \Sigma_{B^{\prime}}^{i}$ in $B \cup_{\partial B}-B^{\prime}$. Using the notation of Section 2.4, we have

$\mathcal{I}_{B B^{\prime}}\left(\beta_{1} \wedge \beta_{2} \wedge \beta_{3}\right)=\left\langle\Sigma_{1}, \Sigma_{2}, \Sigma_{3}\right\rangle_{B \cup_{\partial B}-B^{\prime}}=\left\langle\Sigma_{B}^{1}, \Sigma_{B}^{2}, \Sigma_{B}^{3}\right\rangle_{B}-\left\langle\Sigma_{B^{\prime}}^{1}, \Sigma_{B^{\prime}}^{2}, \Sigma_{B^{\prime}}^{3}\right\rangle_{B^{\prime}}$, where $\left\langle\Sigma_{B}^{1}, \Sigma_{B}^{2}, \Sigma_{B}^{3}\right\rangle_{B}$ is zero, and where $-\left\langle\Sigma_{B^{\prime}}^{1}, \Sigma_{B^{\prime}}^{2}, \Sigma_{B^{\prime}}^{3}\right\rangle_{B^{\prime}}$ identifies directly with $\mu_{123}(L ; M)$. It also follows from the above and a direct computation that $\mathcal{I}_{A A^{\prime}}\left(\alpha_{1} \wedge \alpha_{2} \wedge \alpha_{3}\right)=1$. We thus obtain

$$
\lambda\left(M_{L}\right)-\lambda(M)=-2 \mu_{123}(L)+\lambda\left(S_{L_{\mathrm{st}}}^{3}\right) .
$$

Now, we can use the two lemmas of Section 3 to compute $\lambda\left(S_{L_{\mathrm{st}}}^{3}\right)$ as follows. By applying Lemma $3.3\left|f_{1}\right|+\left|f_{2}\right|+\left|f_{3}\right|$ times to the link $L_{\text {st }}$, we can reduce the framings of its three leaves to zero, and the resulting Borromean surgery link $L^{\prime}$ satisfies

$$
\lambda\left(S_{L_{\mathrm{st}}}^{3}\right)=\lambda\left(S_{L^{\prime}}^{3}\right)-f_{1} \cdot f_{2} \cdot f_{3}+\sum_{\circlearrowleft_{1,2,3}} f_{1} \cdot l_{23} \cdot\left(l_{23}+1\right) .
$$

Next, by suitably applying Lemma $3.4\left|l_{23}\right|+\left|l_{13}\right|+\left|l_{12}\right|$ times to the link $L^{\prime}$, we obtain a Borromean surgery link $L_{f}$ in $S^{3}$ whose leaves are three 0 -framed pairwise unlinked unknots, and such that

$$
\lambda\left(S_{L^{\prime}}^{3}\right)=\lambda\left(S_{L_{f}}^{3}\right)-2 . l_{12} \cdot l_{13} \cdot l_{23} .
$$

Using Kirby calculus, one can easily check that $S_{L_{f}}^{3} \cong S^{3}$ (see for example [2, Section 2.1]). We thus have $\lambda\left(S_{L_{f}}^{3}\right)=0$. It follows that

$$
\lambda\left(S_{L_{\mathrm{st}}}^{3}\right)=-f_{1} \cdot f_{2} \cdot f_{3}-2 \cdot l_{12} \cdot l_{13} \cdot l_{23}+\sum_{\sigma_{1,2,3}} l_{23} \cdot\left(l_{23}+1\right) \cdot f_{1},
$$

which concludes the proof.

\section{Proof of Theorem 2.3}

In order to prove Theorem 2.3, we will use the theory of finite type invariants. 


\subsection{Finite type invariants of integral homology spheres}

The notion of finite type invariants of integral homology spheres is due to Ohtsuki, and is defined using algebraically split, unit-framed links [11]. For the purpose of the present paper, we use an alternative definition using the notion of Borromean surgery, due to Garoufalidis, Goussarov and Polyak [2], Goussarov [3] and Habiro [4].

Let $\mathcal{S}$ denote the free $\mathbf{Z}$-module generated by elements of $\mathbf{Z} H S$. For $k \geq 0$, let $\mathcal{S}_{k}$ denote the $\mathbf{Z}$-submodule of $\mathcal{S}$ generated by elements

$$
[M ; G]:=\sum_{G^{\prime} \subseteq G}(-1)^{\left|G^{\prime}\right|} M_{G^{\prime}}
$$

where $M$ is an integral homology sphere, and where $G=G_{1} \cup \ldots \cup G_{k}$ is a disjoint union of $k$ Borromean surgery links in $M$. The sum runs over all the subsets $G^{\prime}$ of $G$ (regarded as the set of the links $G_{i}$ ) and $\left|G^{\prime}\right|$ denotes the cardinality of $G^{\prime}$. Observe that we have the equality

$$
M_{G}=\sum_{G^{\prime} \subseteq G}(-1)^{\left|G^{\prime}\right|}\left[M ; G^{\prime}\right]
$$

A finite type invariant of degree $k$ is a map $f: \mathbf{Z} H S \longrightarrow A$, where $A$ is an abelian group, whose natural extension to $\mathcal{S}$ vanishes on $\mathcal{S}_{k+1}$.

Proposition 5.1 [11;2] The Casson invariant is a degree 2 finite type invariant.

So we can now use the theory of finite type invariants to study the behavior of the Casson invariant under Borromean surgery.

\subsection{Proof of Theorem 2.3}

Let $L=L_{1} \cup \ldots \cup L_{n}$ be a disjoint union of Borromean surgery links in an integral homology sphere $M$. By using (5-1) and Proposition 5.1, one can check that

$$
\lambda\left(M_{L}\right)-\lambda(M)=\sum_{i=1}^{n}\left(\lambda\left(M_{L_{i}}\right)-\lambda(M)\right)+\sum_{1 \leq i<j \leq n} \lambda\left(\left[M ; Y_{i} \cup Y_{j}\right]\right) .
$$

So we are left with the computation of $\lambda\left(\left[M ; Y_{i} \cup Y_{j}\right]\right)$ for all $i<j$. By Theorem 2.2, we have

$$
\begin{aligned}
\lambda\left(\left[M ; Y_{i} \cup Y_{j}\right]\right) & =\left(\lambda\left(\left(M_{Y_{j}}\right)_{Y_{i}}\right)-\lambda\left(M_{Y_{j}}\right)\right)-\left(\lambda\left(M_{Y_{i}}\right)-\lambda(M)\right) \\
& =-2 \mu_{123}\left(Y_{i} ; M_{Y_{j}}\right)+2 \mu_{123}\left(Y_{i} ; M\right),
\end{aligned}
$$


where we also use the fact that a Borromean surgery preserves the framings and linking numbers [9]. So, if we denote by $\Sigma_{r}$ a Seifert surface for $F_{r}^{i}(r=1,2,3)$, we see that $\lambda\left(\left[M ; Y_{i} \cup Y_{j}\right]\right)$ counts, with signs, the triple intersection points between $\Sigma_{1}, \Sigma_{2}$ and $\Sigma_{3}$ created when doing surgery along $Y_{j}$.

For each leaf $F_{S}^{j}$ of $Y_{j}(s=1,2,3)$, denote by $B_{s}$ the non-leaf component of $Y_{j}$ having linking number 1 with $F_{s}^{j}$, oriented as in Section 2.2. Using Kirby moves, one sees that each positive (resp. negative) intersection point between $\Sigma_{r}$ and $F_{S}^{j}$ $(r, s=1,2,3)$ contributes, via surgery, to a band sum of $F_{r}^{i}$ with a parallel copy of $B_{s}$ with opposite (resp. same) orientation. So surgery along $Y_{j}$ results in band summing copies of $B_{1}, B_{2}$ and $B_{3}$ with the leaves of $Y_{i}$, with the number and orientation of these copies being completely determined by the linking numbers of the leaves of $Y_{i}$ and $Y_{j}$. Each nontrivial contribution to $\mu_{123}$ comes from a triple of band sums of pairwise distinct leaves of $Y_{i}$ with copies of pairwise distinct $B_{s}$ 's. It thus follows from an easy counting argument that

$$
\lambda\left(\left[M ; Y_{i} \cup Y_{j}\right]\right)=-2 \sum_{\sigma \in S_{3}} s(\sigma) l_{1 \sigma(1)}^{i j} l_{2 \sigma(2)}^{i j} l_{3 \sigma(3)}^{i j},
$$

which concludes the proof.

Remark 5.2 The theory of finite type invariants, that we used here to prove Theorem 2.3 , could actually also be used to give an alternative (and quite simple) proof of Theorem 2.2. Such a proof, however, is only valid for integral homology spheres and is therefore not included in this paper.

\section{A remark and an application}

\subsection{Comparison to Lescop's surgery formula}

One can also use Lescop's global surgery formula to express the variation of the Casson invariant under a Borromean surgery, in terms of the multivariable Alexander polynomial $\Delta[6]$.

Consider the Borromean surgery link $L$ as in Figure 1 and denote by $B$ the standard Borromean link. Then $[6,1.4 .8]$ gives that $\lambda\left(M_{L}\right)-\lambda(M)$ equals

$-\left|\begin{array}{lll}f_{1} & l_{12} & l_{13} \\ l_{12} & f_{2} & l_{23} \\ l_{13} & l_{23} & f_{3}\end{array}\right| \cdot \zeta(B)-\sum_{\circlearrowleft_{1,2,3}}\left|\begin{array}{ll}f_{1} & l_{12} \\ l_{12} & f_{2}\end{array}\right| \cdot \zeta\left(B \cup F_{3}\right)-\sum_{\circlearrowleft_{1,2,3}} f_{1} . \zeta\left(B \cup F_{2} \cup F_{3}\right)-\zeta(L)$, 
where, for an $n$-component link $K, \zeta(K)=\partial^{n} /\left(\partial t_{1} \ldots \partial t_{n}\right) \Delta_{K}(1, \ldots, 1)$. For the Borromean link $B$ we know that $\zeta(B)=1$, and one can easily compute the values of $\zeta$ in the two sums of the above formula: $\zeta\left(B \cup F_{i}\right)=0$ and $\zeta\left(B \cup F_{i} \cup F_{j}\right)=-l_{i j}$ for all $1 \leq i \neq j \leq 3$. It follows in particular that

$$
\frac{\partial^{6}}{\partial t_{1} \ldots \partial t_{6}} \Delta_{L}(1, \ldots, 1)=2 . \mu_{123}(L) .
$$

\subsection{An application}

As a conclusion, we give an application of our main theorem. Namely we show how Theorem 2.2 provides an alternative proof of a realization theorem for the Casson invariant of homology spheres of Mazur type.

Recall that a homology sphere of Mazur type is obtained by surgery on $S^{3}$ along a 2-component link $K_{1} \cup K_{2}$ such that $\operatorname{lk}\left(K_{1}, K_{2}\right)= \pm 1, K_{1}$ is a trivial knot with framing 0 and $K_{2}$ has framing $r \in \mathbf{Z}$. Such an integral homology sphere bounds a contractible 4-manifold, and thus its Casson invariant is an even number. Mizuma showed the following.

Theorem 6.1 [10] For any even integer $k$, there is a homology sphere of Mazur type $M$ such that $\lambda(M)=k$.

Mizuma describes this homology sphere in terms of double branched cover of $S^{3}$ along so-called knots of 1-fusion. Theorem 2.2 allows us to give explicit examples in terms of Borromean surgery links. Indeed, consider the link $L_{ \pm n}$ depicted in the left part of Figure 8. There, the three leaves have framing zero. Clearly, by Theorem 2.2, surgery on $S^{3}$ along $L_{ \pm n}$ produces an integral homology sphere with Casson invariant $\mp 2 n$. On the other hand, by Lemma 3.2, the link $L_{ \pm n}$ is Kirby-equivalent to a 2-component link $K_{1} \cup K_{2}$ as depicted on the right-hand side of the figure, which

satisfies the above-mentioned conditions. Therefore $S_{L_{ \pm n}}^{3} \cong S_{K_{1} \cup K_{2}}^{3}$ is a homology sphere of Mazur type.

\section{References}

[1] E Auclair, C Lescop, Clover calculus for homology 3-spheres via basic algebraic topology, Algebr. Geom. Topol. 5 (2005) 71-106 MR2135546

[2] S Garoufalidis, M Goussarov, M Polyak, Calculus of clovers and finite type invariants of 3-manifolds, Geom. Topol. 5 (2001) 75-108 MR1812435 


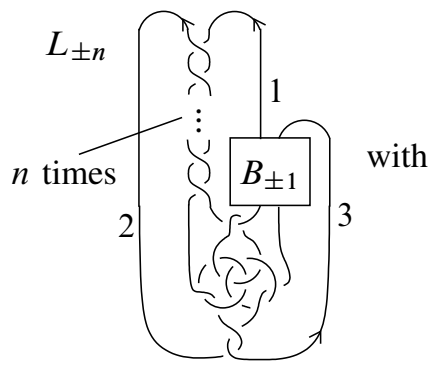

$$
B_{+1}=S
$$$$
B_{-1}=3
$$

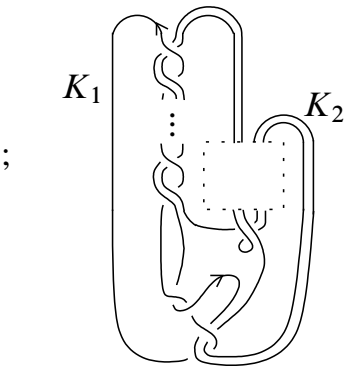

Figure 8

[3] M Goussarov, Finite type invariants and $n$-equivalence of 3-manifolds, C. R. Acad. Sci. Paris Sér. I Math. 329 (1999) 517-522 MR1715131

[4] K Habiro, Claspers and finite type invariants of links, Geom. Topol. 4 (2000) 1-83 MR1735632

[5] J Johannes, A type 2 polynomial invariant of links derived from the Casson-Walker invariant, J. Knot Theory Ramifications 8 (1999) 491-504 MR1697385

[6] C Lescop, Global surgery formula for the Casson-Walker invariant, Annals of Math. Studies 140, Princeton University Press (1996) MR1372947

[7] C Lescop, A sum formula for the Casson-Walker invariant, Invent. Math. 133 (1998) 613-681 MR1645066

[8] G Massuyeau, Spin Borromean surgeries, Trans. Amer. Math. Soc. 355 (2003) 39914017 MR1990572

[9] S V Matveev, Generalized surgeries of three-dimensional manifolds and representations of homology spheres, Mat. Zametki 42 (1987) 268-278, 345 MR915115

[10] Y Mizuma, On the Casson invariant of Mazur's homology spheres, preprint

[11] T Ohtsuki, Finite type invariants of integral homology 3-spheres, J. Knot Theory Ramifications 5 (1996) 101-115 MR1373813

[12] K Walker, An extension of Casson's invariant, Annals of Math. Studies 126, Princeton University Press (1992) MR1154798

CTQM - Department of Mathematical Sciences, University of Aarhus Ny Munkegade, bldg 1530, 8000 Aarhus C, Denmark

meilhan@imf.au.dk

Received: 5 February 2008 Revised: 3 March 2008 